\title{
ON THE ALDER POLYNOMIALS AND A NEW GENERALIZATION OF THE ROGERS-RAMANUJAN IDENTITIES
}

\author{
BY \\ GEORGE E. ANDREWS( $\left.{ }^{1}\right)$
}

\begin{abstract}
In 1954, H. L. Alder obtained certain analy tic generalizations of the Rogers-Ramanujan identities that involved a sequence of polynomials subsequently named the Alder polynomials. The object here is to obtain a new representation of the Alder polynomials from which a new generalization of the Rogers-Ramanujan identities is easily deduced.
\end{abstract}

1. Introduction. In 1954, H. L. Alder [1] proved that there exist polynomials $G_{k, n}(q)$ such that

$$
\prod_{\substack{n=1 \\ n \neq 0, \pm k(\bmod 2 k+1)}}^{\infty}\left(1-q^{n}\right)^{-1}=\sum_{n=0}^{\infty} G_{k, n}(q) /(q)_{n},
$$

and

$$
\prod_{\substack{n=1 \\ n \neq 0, \pm 1(\bmod 2 k+1)}}^{\infty}\left(1-q^{n}\right)^{-1}=\sum_{n=0}^{\infty} G_{k, n}(q) q^{n} /(q)_{n},
$$

where $(a)_{n}=(a ; q)_{n}=(1-a)(1-a q) \cdots\left(1-a q^{n-1}\right),(a)_{0}=1$, and $(a)_{\infty}=$ $\lim _{n \rightarrow \infty}(a)_{n}$. As Alder remarks $G_{2, n}(q)=q^{n^{2}}$, and so when $k=2$ the above identities reduce to the celebrated Rogers-Ramanujan identities.

As is well known (cf. [8, pp. 290-291]), the Rogers-Ramanujan identities imply two partition identities.

The number of partitions of $n$ with minimal difference at least 2 between parts equals the number of partitions of $n$ into parts congruent to \pm 1 modulo 5 .

Received by the editors August 10, 1972.

AMS (MOS) subject classifications (1970). Primary 10A45; Secondary 05A19, 33A30.

Key words and phrases. Partitions, partition identities, Alder polynomials, RogersRamanujan identities.

(1) Partially supported by National Science Foundation Grant GP-23774. The results in this paper were presented in the Special Session on Number Theory at the 701 st meeting of the Society in Dallas, Texse on January 26, 1973.

The author expresses many thanks to a very helpful referee. 
The number of partitions of $n$ into parts each greater than 1 with (1.4) minimal difference at least 2 between parts equals the number of partitions of $n$ into parts congruent to \pm 2 modulo 5 .

Alder remarks [1, p. 162] that he has been unable to interpret partitiontheoretically the right-hand side of (1.1) or (1.2) except for the known case $k=$ 2 given above. V. N. Singh in [10], [11] and [12] and L. Carlitz in [6] have given further properties of these polynomials. In $[2, \S 6]$, Alder gives a brief survey of the results known about these polynomials, and again he mentions that (1.1) and (1.2) have not yielded partition-theoretic interpretations. In 1961, B. Gordon [7] gave the following "partition-theoretic" generalization of the Rogers-Ramanujan identities.

The number of partitions of $n$ into parts not congruent to 0 , $\pm t(\bmod 2 k+1)$, where $1 \leqslant t \leqslant k$, is equal to the number of partitions of $n$ of the form $b_{1}+b_{2}+\cdots+b_{n}$, where $b_{i} \geqslant$ $b_{i+1}, b_{i} \geqslant b_{i+k-1}+2$, and $b_{h-t+1} \geqslant 2$.

But Gordon remarks [7, p. 394] that while (1.5) suggests that it should be possible to interpret the right-hand side of (1.1) and (1.2) partition-theoretically, he is unable to give such an interpretation.

The object of this paper is to provide a new partition-theoretic generalization of the Rogers-Ramanujan identities which arises from interpreting (1.1), (1.2) (and $k-2$ related identities). Surprisingly the new generalization relates partitions into parts not congruent to $0, \pm t(\bmod 2 k+1)$ to certain restricted partitions that are completely different from those described in (1.5). In fact, the interpretation yielded from (1.1) and (1.2) in the case $k=2$ is not (1.3) and (1.4) but the following:

The number of partitions of $n$ in which each part is at least as large (1.6) as the number of parts equals the number of partitions of $n$ into parts congruent to \pm 1 modulo 5 .

The number of partitions of $n$ in which each part is larger than the (1.7) number of parts equals the number of partitions of $n$ into parts congruent to \pm 2 modulo 5 .

Identities (1.6) and (1.7) are easily derived from (1.1) and (1.2) in the case $k=2$; just follow the derivation of (1.3) and (1.4) given in [8, §19.13] except 
for (1.6) use the identity $m^{2}=m+m+\cdots+m$ rather than $m^{2}=1+3+$ $\cdots+(2 m-1)$ and for (1.7) use the identity $m^{2}+m=(m+1)+(m+1)+$ $\cdots+(m+1)$ rather than $m^{2}+m=2+4+\cdots+2 m$. However, it is not at all clear how to relate our general result to that of Gordon except in the simple case $k=2$.

The next section will be devoted to definitions and stating our main results. In $\S 3$, we prove some lemmas on certain partition generating functions. In $\S 4$, we prove our main results. $\$ 5$ is devoted to a brief discussion of the Alder polynomials for $k=3$, where four different representations of these polynomials are discussed. The concluding section is devoted to questions raised by the results proved here.

2. Definitions and theorem statements.

Definition 1 . Let $k, i, j, l, N$ and $m$ be integers satisfying $0 \leqslant i \leqslant$ $k+1,0 \leqslant l \leqslant k-1(j, m$ and $N$ are unrestricted integers and may be positive, negative or zero). We define $p_{k, i}(j ; l ; N ; m)$ to be the number of partitions of $m$ into exactly $j$ parts of the form $m=b_{1}+b_{2}+\cdots+b_{j}$, where each $b_{i}$ is $\leqslant N$, at most $l$ of the $b$ 's equal $N, b_{s} \geqslant b_{s+1}, b_{s}-b_{s+k-1} \geqslant$ 2 , and at most $i-1$ of the $b$ 's are equal to 1 .

There are several important points to observe about the partition function defined above. First since the empty partition of 0 is the only partition with 0 parts, we see that for $1 \leqslant i \leqslant k+1,0 \leqslant l \leqslant k-1$,

$$
p_{k, i}(0 ; l ; N ; m)= \begin{cases}1 & \text { if } m=0 \\ 0 & \text { if } m \neq 0\end{cases}
$$

Next if $i=0$, we see the condition that 1 appears at most $i-1$ times is violated for every partition, so

$$
p_{k, 0}(t ; l ; N ; m)=0 .
$$

Next we observe that there exists only one partition in which there is a nonpositive number of parts, namely the empty partition of zero. Also there exists only one partition of a nonpositive integer, again the empty partition of 0 . Therefore

$$
p_{k, i}(j ; l ; N ; m)=0 \text { if } m \leqslant 0 \text { or } j \leqslant 0 \text { and } j^{2}+m^{2} \neq 0 \text {. }
$$

Finally since no part of a partition can exceed the number being partitioned, we see that by Gordon's theorem ((1.5) of $\S 1)$ :

$$
\sum_{m=0}^{\infty} \sum_{j=0}^{\infty} p_{k, i}(j ; k-1 ; m ; m) q^{m}=\prod_{\substack{n=1 ; \\ n \neq 0, \pm i(\bmod 2 k+1)}}^{\infty}\left(1-q^{n}\right)^{-1}
$$

Definition 2. We let $D_{k, l}(j ; l ; N ; q)$ denote the generating function for 
$p_{k, i}(j ; l ; N ; m)$. By this we mean

$$
\begin{aligned}
D_{k, i}(j ; l ; N ; q) & =\sum_{m=-\infty}^{\infty} p_{k, l}(j ; l ; N ; m) q^{m} \\
& =\sum_{m=0}^{\infty} p_{k, i}(j ; l ; N ; m) q^{m} .
\end{aligned}
$$

While our first two definitions are closely related to the restricted partitions described in (1.5), the following definition provides a completely new parameter for partitions.

Definition 3. Suppose that $\pi$ is a partition of $m$ into $n$ parts. Let $j$ be the smallest integer with $0 \leqslant j \leqslant n$ such that the largest $n-j$ parts of $\pi$ are each $\geqslant[(n+j+2 k-i-2) /(k-1)]$ where $k>1$, and $[x]$ is the largest integer $\leqslant x$. We say that $[(n+j+k-i-2) /(k-1)]$ is the $(k, i)$ cutoff of $\pi$.

Clearly such a $j$ exists, since the inequality in question is vacuously satisfied with $j=n$. We note that since the $(k, i)$-cutoff is $[(n+j+k-i-2) /(k-1)]$ where $j$ is the smallest integer in $[0, n]$ such that the largest $n-j$ parts of $\pi$ are $\geqslant[(n+j+2 k-i-2) /(k-1)]$, the remaining $j$ parts are

$$
\leqslant\left[\frac{n+(j+1)+2 k-i-2}{k-1}\right]-1=\left[\frac{n+j+k-i-2}{k-1}\right],
$$

the $(k, i)$-cutoff.

Definition 4. We define $Q_{k, i}(z ; q)$ by

$$
\sum_{n=0}^{\infty} \frac{(-1)^{n} z^{k n} q^{1 / 2(2 k+1) n(n+1)-i n}\left(1-z^{i} q^{(2 n+1) t}\right)}{(q)_{n}\left(z q^{n+1}\right)_{\infty}}=Q_{k, i}(z ; q) .
$$

We remark that Selberg [9] studied the function $C_{k, i}(z ; q)=(z q)_{\infty} Q_{k, i}(z ; q)$, and he showed that

$$
Q_{k, 0}(z ; q)=0
$$

and

$$
Q_{k, i}(z ; q)-Q_{k, i-1}(z ; q)=z^{i-1} q^{i-1} Q_{k, k-i+1}(z q ; q) .
$$

DEFINITION 5. We define $G_{k, i}(n ; q)$ by

$$
Q_{k, i}(z ; q)=\sum_{n=0}^{\infty} G_{k, i}(n ; q) z^{n} /(q)_{n} .
$$

We shall refer to the $G_{k, i}(n ; q)$ as "Alder polynomials". While Alder [1] only considered in detail $G_{k, k}(n ; q)$ (which he called $G_{k, n}(q)$ ), he makes clear 
at the end of his paper that one can find such polynomials for $1 \leqslant i \leqslant k$. It is not immediately obvious that the $G_{k, i}(n ; q)$ are polynomials in $q$; however, we shall establish recurrence formulae in $\S 3$ that are both necessary for further developments and do establish that the $G_{k, i}(n ; q)$ are polynomials.

DEFINITION 6. Let

$$
\eta(m)=\eta_{k}(m)= \begin{cases}r+1 & \text { if } m \equiv r(\bmod k-1), 0 \leqslant r \leqslant k-3, \\ k-2 & \text { if } m \equiv k-2(\bmod k-1) .\end{cases}
$$

We are now in a position to state our fundamental theorem on the Alder polynomials.

THEOREM 1. For $1 \leqslant i \leqslant k, k \geqslant 2$,

$$
\begin{aligned}
G_{k, i}(n ; q)= & \sum_{j=0}^{\infty} q^{(n-j)\left(\frac{(n+j+2 k-i-2)}{(k-1)}\right)}\left(1-q^{n}\right) \cdots \\
& \left(1-q^{n-j+1}\right) D_{k-1, i}\left(j ; \eta(n+j+k-i-2) ;\left[\frac{n+j+k-i-2}{k-1}\right] ; q\right) .
\end{aligned}
$$

Apart from the partition-theoretic usefulness of Theorem 1, we see that it also establishes the polynomial nature of $G_{k, i}(n ; q)$. The next theorem is an easy consequence of Theorem 1 .

THEOREM 2. Let $A_{k, i}(n)$ denote the number of partitions of $n$ into parts not congruent to $0, \pm i$ modulo $2 k+1$. Let $H_{k, i}(n)$ denote the number of partitions of $n$ in which the $j$ parts that do not exceed the $(k, i)$-cutoff when taken together form a partition of the type generated by

$$
D_{k-1, i}\left(j ; \eta(n+j+k-i-2) ;\left[\frac{(n+j+k-i-2)}{(k-1)}\right] ; q\right) .
$$

Then $A_{k, i}(n)=H_{k, i}(n)$.

This result certainly resembles Gordon's theorem (1.5) in that $A_{k, i}(n)$ appears in both theorems. However, the partitions enumerated by $H_{k, i}(n)$ are distantly related to the restricted partitions considered by Gordon. For one of the partitions (say $b_{1}+b_{2}+\cdots+b_{s}$ ) considered by Gordon, we have $b_{j} \geqslant$ $b_{j+1}, b_{j}-b_{j+k-1} \geqslant 2$, and at most $i-1$ ones appear. For the partitions of the type enumerated by $H_{k, i}(n)$ (say $c_{1}+c_{2}+\cdots+c_{s}$ ) we have no restrictions for parts that exceed the $(k, i)$-cutoff. However, the actual $(k, i)$-cutoff can appear at most once more than the least nonnegative residue of $n+j-i-2$ modulo $k-1$, and parts not exceeding the $(k, i)$-cutoff satisfy Gordon's conditions at the $(k-1)$ level, namely $c_{j} \geqslant c_{j+1}, c_{j}-c_{j+k-2} \geqslant 2$, and at most $i-1$ ones appear. 
At the end of $\S 4$ we shall show how to derive (1.6) and (1.7) from Theorem 2 in the case $k=2, i=2,1$. At that time we shall give some examples contrasting the types of partitions arising in Gordon's theorem and those arising in Theorem 2.

3. Lemmas for the $D_{k, i}(j ; l ; N ; q)$. Our work on the Alder polynomials will depend on certain recurrence formulas for the $D_{k, i}(j ; l ; N ; q)$ which we shall develop here.

Lemma 1. For $1 \leqslant i \leqslant k+1,0 \leqslant l \leqslant k-1$, and for all integers $j$ and $N$,

$$
D_{k, i}(j ; l ; N ; q)-D_{k, i-1}(j ; l ; N ; q)=q^{j} D_{k, k-i+1}(j-i+1 ; l ; N-1 ; q) .
$$

Proof. We first treat the nontrivial case when $j>0, N>0,1 \leqslant i \leqslant k$. We shall prove the equivalent identity for the related partition functions. Namely,

$$
\begin{aligned}
p_{k, i}(j ; l ; N ; m)-p_{k, i-1}(j ; l ; N ; m) \\
\quad=p_{k, k-i+1}(j-i+1 ; l ; N-1 ; m-j) .
\end{aligned}
$$

The left-hand side of (3.1) enumerates those partitions $b_{1}+b_{2}+\cdots+b_{j}$ of $m$ into exactly $j$ parts each $\leqslant N$ that satisfy $b_{h} \geqslant b_{h+1}, b_{h}-b_{h+k-1} \geqslant$ 2, exactly $i-1$ ones appear, and $N$ appears at most $l$ times. If we delete all the ones and subtract 1 from the remaining summands we find that we now have a partition of $m-j$ into $j-i+1$ parts where now no part exceeds $N-$ 1 and $N-1$ appears at most $l$ times. Also since originally 2 could appear at most $(k-1)-(i-1)$ times, now 1 appears at most $(k-i+1)-1$ times. Since the transformed partitions still satisfy the original difference conditions on the parts we see that we now have a partition of the type enumerated by $p_{k, k-i+1}(j-i+1 ; l ; N-1 ; m-j)$. Since the above process is clearly reversible, we see that we have established a one-to-one correspondence between the two types of partitions being considered. Thus (3.1) is established for $j>0$ and $N>0$.

If $i=k+1$, then the restriction that at most $k$ ones appear is equivalent to the restriction that at most $k-1$ ones appear since the difference conditions on the parts prohibit any parts from appearing more than $k-1$ times. Hence in the case $i=k+1$, the left side of (3.1) is identically 0 as is the right-hand side since $p_{k, 0}(j-k+1 ; l ; N-1 ; m-j)=0$.

If $j=0$ and $i \geqslant 2$, then $j-i+1<0$; in this case (3.1) reduces to $1-1=0$. If $j=0$ and $i=1$ then (3.1) reduces to the identity $1-0=1$. If $j \leqslant 0$, (3.1) reduces to $0-0=0$. If $N \leqslant 0$ and $j \neq 0$, (3.1) reduces to $0-0=0$. Thus we see that (3.1) is valid in all cases under consideration, and therefore Lemma 1 is established. 
LEMMA 2. For $1 \leqslant i \leqslant k$,

$$
D_{k, i}(j ; 0 ; N ; q)=D_{k, i}(j ; k-1 ; N-1 ; q) \text {. }
$$

Proof. The condition on the partition $b_{1}+b_{2}+\cdots+b_{j}$ that $b_{h} \geqslant$ $b_{h+1}$ and $b_{h}-b_{h+k-1} \geqslant 2$ implies that no part appears more than $k-1$ times. The restriction that no part exceed $N$ and that $N$ appears at most 0 times is clearly equivalent to the condition that no part exceed $N-1$, and $N-1$ occurs at most $k-1$ times.

Lemma 3. For $1 \leqslant i \leqslant k, 1 \leqslant l \leqslant k-1$,

$$
D_{k, i}(j ; l ; N ; q)=D_{k, i}(j ; l-1 ; N ; q)+q^{l N} D_{k, i}(j-l ; k-1-l ; N-1 ; q) .
$$

Proof. We shall prove the equivalent identity

$$
\begin{aligned}
& p_{k, i}(j ; l ; N ; m) \\
& \quad=p_{k, l}(j ; l-1 ; N ; m)+p_{k, l}(j-l ; k-1-l ; N-1 ; m-l N) .
\end{aligned}
$$

If $N \geqslant 2$, we may prove (3.2) as follows: We split the partitions enumerated by the left side of (3.2) into two classes: (a) those in which $N$ appears fewer than $l$ times, and (b) those in which $N$ appears exactly $l$ times. The partitions in class (a) are clearly enumerated by $p_{k, i}(j ; l-1 ; N ; m)$. We delete all the $N$ s from the partitions in the second class. Since $N \geqslant 2$, this means that $N-$ 1 can appear at most $k-1-l$ times due to the difference conditions on the parts. The number of parts is now reduced to $j-l$, and the number being partitioned is $m-N l$. Hence since the above process is reversible, the class (b) is in one-to-one correspondence with the partitions enumerated by $p_{k, i}(j-l ; k-1-l ; N-1 ; m-l N)$. Thus (3.2) is established if $N \geqslant 2$.

If $N=1$, then

$$
\begin{aligned}
p_{k, i}(j ; l ; 1 ; m) & = \begin{cases}1 & \text { if } 0 \leqslant j=m \leqslant \min (i-1, l), \\
0 & \text { otherwise, }\end{cases} \\
p_{k, i}(j ; l-1 ; 1 ; m) & = \begin{cases}1 & \text { if } 0 \leqslant j=m \leqslant \min (i-1, l-1), \\
0 & \text { otherwise, }\end{cases} \\
p_{k, i}(j-l ; k-1-l ; 0 ; m-l) & = \begin{cases}1 & \text { if } j=l=m, \\
0 & \text { otherwise. }\end{cases}
\end{aligned}
$$

Thus the only instance for $N=1$ in which $p_{k, i}(j ; l ; 1 ; m) \neq p_{k, i}(j ; l-1 ; 1 ; m)$ is the case $j=l=m$ and in this instance (3.2) reduces to $1=0+1$. Thus (3.2) is valid for $N=1$.

For $N \leqslant 0$, we see that (3.2) reduces to $1=1+0$ if $j=m=0$ and to $0=0+0$ otherwise. Hence (3.2) is completely established. 
4. Proof of theorems. Our first object is to prove Theorem 1. We recall Definitions 4 and 5 for the identity

$$
Q_{k, i}(z ; q)=\sum_{n=0}^{\infty} G_{k, i}(n ; q) z^{n} /(q)_{n}, \quad 1 \leqslant i \leqslant k .
$$

Since $Q_{k, i}(0 ; q)=1$, we see that

$$
G_{k, i}(n ; q)= \begin{cases}1 & \text { if } n=0 \\ 0 & \text { if } n<0\end{cases}
$$

From (2.6) we deduce that for $2 \leqslant i \leqslant k$,

$$
\begin{aligned}
G_{k, i}(n ; q) & -G_{k, i-1}(n ; q) \\
& =q^{n}\left(1-q^{n}\right) \cdots\left(1-q^{n-i+2}\right) G_{k, k-i+1}(n-i+1 ; q) .
\end{aligned}
$$

Combining (2.5) with (2.6) in the case $i=1$, we see that

$$
G_{k, 1}(n ; q)=q^{n} G_{k, k}(n ; q) .
$$

We remark now that equations (4.1), (4.2) and (4.3) uniquely define the $G_{k, i}(n ; q)$ and establish the fact that the $G_{k, i}(n ; q)$ are polynomials in $q$. The proof of this assertion is by induction on $n$. The assertion is obvious for $n=0$. For general $n$, we add the $k-1$ different equations given by (4.2); this yields

$$
\begin{aligned}
G_{k, k}(n ; q) & -G_{k, 1}(n ; q) \\
= & \sum_{i=2}^{k} q^{n}\left(1-q^{n}\right) \cdots\left(1-q^{n-i+2}\right) G_{k, k-i+1}(n-i+1 ; q) .
\end{aligned}
$$

Replacing $G_{k, 1}(n ; q)$ by $q^{n} G_{k, k}(n ; q)$ in (4.4), we see that

$$
G_{k, k}(n ; q)=\sum_{i=2}^{k} q^{n}\left(1-q^{n-1}\right) \cdots\left(1-q^{n-i+2}\right) G_{k, k-i+1}(n-i+1 ; q) .
$$

Hence by the induction hypothesis the right-hand side is uniquely defined and is a polynomial in $q$. Therefore the same is true of $G_{k, k}(n ; q)$, and by (4.3) the same is true of $G_{k, 1}(n ; q)$. For each $i_{0}$ with $2 \leqslant i_{0}<k$, we add the $i_{0}-1$ equations defined by (4.2) with $i=2,3, \cdots, i_{0}$. This addition produces

$$
G_{k, i_{0}}(n ; q)=G_{k, 1}(n ; q)+\sum_{i=2}^{i_{0}} q^{n}\left(1-q^{n-1}\right) \cdots
$$

$$
\left(1-q^{n-i+2}\right) G_{k, k-i+1}(n-i+1 ; q) .
$$

Thus $G_{k, i_{0}}(n ; q)$ is also uniquely defined and is a polynomial in $q$. Hence, (4.1), (4.2) and (4.3) do indeed uniquely define the Alder polynomials $G_{k, i}(n ; q)$, $1 \leqslant i \leqslant k$. 
We now define an apparently new set of polynomials

$$
\begin{aligned}
g_{k, i}(n ; q)= & \sum_{j=0}^{n} q^{(n-j) ! \frac{n+j+2 k-i-2}{k-1} \mid}\left(1-q^{n}\right) \cdots \\
& \quad\left(1-q^{n-j+1}\right) D_{k-1, i}\left(j ; \eta(n+j+k-i-2) ;\left[\frac{n+j+k-i-2}{k-1}\right] ; q\right) .
\end{aligned}
$$

We shall establish that these are the Alder polynomials (which is the assertion of Theorem 1) by showing that they too satisfy (4.1), (4.2) and (4.3).

If $k=2$, then we note that for $i=1,2$,

$$
D_{1, i}(j ; l ; N ; q)=\left\{\begin{array}{cc}
1 & \text { if } j=0 \\
0 & \text { otherwise }
\end{array}\right.
$$

because the empty partition of zero (which has zero parts) is the only partition ever enumerated by this generating function. Consequently when $k=2$, equation (4.7) reduces to

$$
\begin{aligned}
g_{2, i}(n ; q) & =q^{n(n+2-i)} \\
& =\left\{\begin{array}{l}
q^{n^{2}} \text { for } i=2, \\
q^{n^{2}+n} \text { for } i=1
\end{array}\right.
\end{aligned}
$$

But this identity together with the Rogers-Ramanujan identities shows that $g_{2, i}(n ; q)=G_{2, i}(n ; q)$ for $i=1,2$. In the following we shall therefore assume that $k \geqslant 3$.

Clearly $g_{k, i}(0 ; q)=1$ since $D_{k, i}(0 ; l ; N ; q)=1$, and $g_{k, i}(n ; q)=0$ for $n<0$ since the sum is empty. Therefore the $g_{k, i}(n ; q)$ satisfy $(4.1)$.

The proof of (4.3) is also simple. In this case

$$
\begin{aligned}
g_{k, 1}(n ; q)= & \sum_{j=0}^{n} q^{(n-j)\left[\frac{n+j+2 k-3}{k-1}\right]}\left(1-q^{n}\right) \cdots \\
& \left(1-q^{n-j+1}\right) D_{k-1,1}\left(j, \eta(n+j+k-3) ;\left[\frac{n+j+k-3}{k-1}\right] ; q\right) \\
= & \sum_{j=0}^{n} q^{(n-j)\left(\left[\frac{n+j+k-2}{k-1}\right]+1\right)}\left(1-q^{n}\right) \cdots \\
& \left(1-q^{n-j+1}\right) D_{k-1,1}\left(j ; \eta(n+j-2) ;\left[\frac{n+j-2}{k-1}\right]+1 ; q\right)
\end{aligned}
$$




$$
\begin{aligned}
& =\sum_{j=0}^{n} q^{(n-j)\left(\left[\frac{n+j+k-2}{k-1}\right]+1\right)}\left(1-q^{n}\right) \cdots \\
& \left(1-q^{n-j+1}\right) q^{j} D_{k-1, k-1}\left(j ; \eta(n+j-2) ;\left[\frac{n+j-2}{k-1}\right] ; q\right) \\
& \text { (by Lemma 1) } \\
& =q^{n} \sum_{j=0}^{n} q^{(n-j)\left[\frac{n+j+k-2}{k-1}\right]}\left(1-q^{n}\right) \cdots \\
& \left(1-q^{n-j+1}\right) D_{k-1, k}\left(j ; \eta(n+j-2) ;\left[\frac{n+j-2}{k-1}\right] ; q\right) \\
& \text { (since } D_{k-1, k-1}=D_{k-1, k} \text { ) } \\
& =q^{n} g_{k, k}(n ; q) .
\end{aligned}
$$

The proof of (4.2) is our hardest task. We must treat for $2 \leqslant i \leqslant k$,

$$
\begin{aligned}
& g_{k, i}(n ; q)-g_{k, i-1}(n ; q)=\sum_{i=0}^{n} q^{(n-i) ! \frac{n+i+2 k-i-2}{k-1}-1}\left(1-q^{n}\right) \cdots \\
&\left(1-q^{n-i+1}\right) D_{k-1, i}\left(j ; \eta(n+j+k-i-2) ;\left[\frac{n+j+k-i-2}{k-1}\right] ; q\right) \\
&- \sum_{j=0}^{n} q^{(n-i) ! \frac{n+i+2 k-i-1}{k-1}}\left(1-q^{n}\right) \cdots \\
&\left(1-q^{n-i+1}\right) D_{k-1, i-1}\left(j ; \eta(n+j+k-i-1) ;\left[\frac{n+j+k-i-1}{k-1}\right]: q\right) .
\end{aligned}
$$

We note that the upper index in each sum is now $n$. This upper index can be replaced by $+\infty$ since for $j>n$, the product $\left(1-q^{n}\right) \cdots\left(1-q^{n-j+1}\right)$ contains the term $\left(1-q^{0}\right)=0$. Next we assert that the lower index in each sum (now 0 ) may be replaced by $\rightarrow$ since for $j<0, D_{k-1, i}(j ; l ; N ; q)=0$.

We now replace $\Sigma_{j=-\infty}^{\infty} T_{j}$ by $\Sigma_{s=-\infty}^{\infty} \Sigma_{t=0}^{k-2} T_{(k-1) s+t+i-r-1}$ where $r$ is defined by $n=(k-1) m+r, 0 \leqslant r<k-1$. Thus we may rewrite (4.8) as $g_{k, i}(n ; q)-g_{k, i-1}(n ; q)$

$$
\begin{aligned}
= & \sum_{i=(k=-1) s+\infty}^{\infty} \sum_{t=0}^{k-2} q^{(n-i)(m+r-1} q^{\left(m+s+2+\left(\frac{t-1}{k-1}\right)\right.}\left(1-q^{n}\right) \cdots \\
& \left(1-q^{n-i+1}\right) D_{k-1, i}\left(j ; \eta(t-2) ; m+s+1+\left[\begin{array}{c}
t-2 \\
k-1
\end{array}\right]: q\right) .
\end{aligned}
$$

$$
\begin{aligned}
&-\sum_{\substack{s=-\infty \\
k=1) s+t+i=r-1}} \sum_{t=0}^{k-2} q^{(n-i)\left(m+s+2+1-\frac{t}{k-1} \mid\right)}\left(1-q^{n}\right) \cdots \\
&\left(1-q^{n-i+1}\right) D_{k-1, i-1}\left(j ; \eta(t-1) ; m+s+1+\left[\begin{array}{c}
t-1 \\
k-1
\end{array}\right]: q\right) .
\end{aligned}
$$


We now split the $\Sigma_{t=0}^{k-2}$ sum into three parts according to $t=0, t=1$ and $t \geqslant 2$. To simplify notation we shall write $j=j(s, t)=(k-1) s+t+i-r-1$. We also note that $\left(1-q^{n}\right) \cdots\left(1-q^{n-j+1}\right)=\left(q^{n-j+1}\right)_{\infty} /\left(q^{n+1}\right)_{\infty}$, so that this expression is well defined if $n \geqslant 0$ and $-\infty<j<\infty$. Hence

$$
\begin{aligned}
& g_{k, i}(n ; q)-g_{k, i-1}(n ; q) \\
& =\sum_{s=-\infty ; j=j(s, 0)}^{\infty} q^{(n-i)(m+s+1)} \frac{\left(q^{n-j+1}\right)_{\infty}}{\left(q^{n+1}\right)_{\infty}} D_{k-1, i}(j ; k-2 ; m+s ; q) \\
& +\sum_{s=-\infty ; j=j(s, 1)}^{\infty} q^{(n-j)(m+s+2)\left(q^{n-j+1}\right)_{\infty}} D_{k-1, i}(j ; k-2 ; m+s ; q)
\end{aligned}
$$

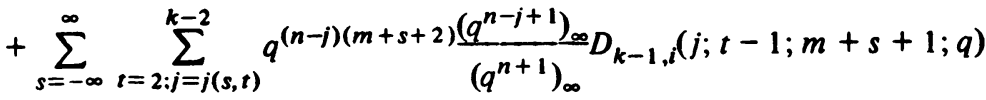

$$
\begin{aligned}
& -\sum_{s=-\infty ; j=j(s, 0)}^{\infty} q^{(n-j)(m+s+2)} \frac{\left(q^{n-j+1}\right)_{\infty}}{\left(q^{n+1}\right)_{\infty}} D_{k-1, i-1}(j ; k-2 ; m+s ; q) \\
& -\sum_{s=-\infty ; j=j(s, 1)}^{\infty} q^{(n-j)(m+s+2) \frac{\left(q^{n-j+1}\right)_{\infty}}{\left(q^{n+1}\right)_{\infty}} D_{k-1, i-1}(j ; 1 ; m+s+1 ; q)} \\
& -\sum_{s=-\infty}^{\infty} \sum_{t=2: j=j(s, t)}^{k-2} q^{(n-j)(m+s+2)} \frac{\left(q^{n-j+1}\right)_{\infty}}{\left(q^{n+1}\right)_{\infty}} D_{k-1, i-1}(j ; t ; m+s+1 ; q) .
\end{aligned}
$$

We combine the first sum with the fourth, the second sum with the fifth, and the third with the sixth. This yields

$$
\begin{aligned}
& g_{k, i}(n ; q)-g_{k, i-1}(n ; q) \\
& =\sum_{\substack{s=-\infty \\
j=j(s, 0)}}^{\infty} q^{(n-j)(m+s+1)} \frac{\left(q^{n-j+1}\right)_{\infty}}{\left(q^{n+1}\right)_{\infty}}\left\{D_{k-1, i}(j ; k-2 ; m+s ; q)\right. \\
& \left.-q^{n-i} D_{k-1, i-1}(j ; k-2 ; m+s ; q)\right\} \\
& \text { (4.11) } \quad+\sum_{\substack{s=-\infty ; \\
j=j(s, i)}}^{\infty} q^{(n-j)(m+s+2)} \frac{\left(q^{n-j+1}\right)_{\infty}}{\left(q^{n+1}\right)_{\infty}}\left\{D_{k-1, i}(j ; k-2 ; m+s ; q)\right. \\
& \left.-D_{k-1, i-1}(j ; 1 ; m+s+1 ; q)\right\} \\
& +\sum_{s=-\infty}^{\infty} \sum_{\substack{t=2 \\
j=j(s, t)}}^{k-2} q^{(n-j)(m+s+2)} \frac{\left(q^{n-j+1}\right)_{\infty}}{\left(q^{n+1}\right)_{\infty}}\left\{D_{k-1, i}(j ; t-1 ; m+s+1 ; q)\right. \\
& \left.-D_{k-1, i-1}(j ; t ; m+s+1 ; q)\right\} .
\end{aligned}
$$


The expressions inside the curly brackets can now be transformed by means of Lemmas 1,2 and 3. We obtain

$$
\begin{aligned}
& D_{k-1, i}(j ; k-2 ; m+s ; q)-q^{n-i} D_{k-1, i-1}(j ; k-2 ; m+s ; q) \\
&=\left(1-q^{n-j}\right) D_{k-1, t-1}(j ; k-2 ; m+s ; q) \\
&+q^{j} D_{k-1, k-i}(j-i+1 ; k-2 ; m+s-1 ; q), \\
& D_{k-1, i}(j ; k-2 ; m+s ; q)-D_{k-1, i-1}(j ; 1 ; m+s+1 ; q) \\
&=q^{j} D_{k-1, k-i}(j-i+1 ; k-2 ; m+s-1 ; q) \\
& \quad-q^{m+s+1} D_{k-1, i-1}(j-1 ; k-3 ; m+s ; q),
\end{aligned}
$$

and

$$
\begin{aligned}
D_{k-1, i} & (j ; t-1 ; m+s+1 ; q)-D_{k-1, t-1}(j ; t ; m+s+1 ; q) \\
= & q^{j} D_{k-1, k-i}(j-i+1 ; t-1 ; m+s ; q) \\
& -q^{t(m+s+1)} D_{k-1, i-1}(j-t ; k-2-t ; m+s ; q) .
\end{aligned}
$$

Substituting the above three identities in (4.11), we see that

$$
\begin{aligned}
& g_{k, i}(n ; q)-g_{k, t-1}(n ; q) \\
& =\sum_{\substack{s=-\infty \\
j=j(s, 0)}}^{\infty} q^{(n-j)(m+s+1) \frac{\left(q^{n-j}\right)_{\infty}}{\left(q^{n+1}\right)_{\infty}} D_{k-1, i-1}(j ; k-2 ; m+s ; q)} \\
& +\sum_{\substack{s=-\infty \\
j=j(s, 0)}}^{\infty} q^{(n-j)(m+s+1)+j} \frac{\left(q^{n-j+1}\right)_{\infty}}{\left(q^{n+1}\right)_{\infty}} D_{k-1, k-i}(j-i+1 ; k-2 ; m+s-1 ; q) \\
& +\sum_{\substack{s=-\infty \\
j=j(s, 1)}}^{\infty} q^{(n-j)(m+s+2)+\frac{\left(q^{n-j+1}\right)_{\infty}}{\left(q^{n+1}\right)_{\infty}}} D_{k-1, k-i}(j-i+1 ; k-2 ; m+s-1 ; q) \\
& -\sum_{\substack{s=-\infty \\
j=j(s, i)}}^{\infty} q^{(n-i)(m+s+2)+(m+s+1) \frac{\left(q^{n-i+1}\right)_{\infty}}{\left(q^{n+1}\right)_{\infty}} D_{k-1, i-1}(j-1 ; k-3 ; m+s ; q)} \\
& +\sum_{s=-\infty}^{\infty} \sum_{\substack{t=2 ; \\
j=j(s, i)}}^{k-2} q^{(n-i)(m+s+2)+\frac{\left(q^{n-j+1}\right)_{\infty}}{\left(q^{n+1}\right)_{\infty}} D_{k-1, k-i}(j-i+1 ; t-1 ; m+s ; q)} \\
& -\sum_{s=-\infty}^{\infty} \sum_{\substack{t=2 ; \\
j=j(s, t)}}^{k-2} q^{(n-j)(m+s+2)+t(m+s+1)} \frac{\left(q^{n-j+1}\right)_{e}}{\left(q^{n+1}\right)_{\infty}} \\
& \cdot D_{k-1, t-1}(j-t ; k-2-t ; m+s ; q) .
\end{aligned}
$$


We now use Lemmas 1 and 3 to transform the $D_{k-1, i-1}(j ; k-2 ; m+s ; q)$ in the first sum appearing in (4.12). Namely

$$
\begin{aligned}
& D_{k-1, i-1}(j ; k-2 ; m+s ; q) \\
& \quad=D_{k-1, i-1}(j ; k-3 ; m+s ; q) \\
& \quad+q^{(k-2)(m+s)} D_{k-1, i-1}(j-k+2 ; k-2 ; m+s-2 ; q)
\end{aligned}
$$

(by Lemmas 2 and 3)

$$
\begin{aligned}
= & D_{k-1, i-1}(j ; k-3 ; m+s ; q) \\
& +q^{(k-2)(m+s)-j+i-2} D_{k-1, k-i+1}(j-i+2 ; k-2 ; m+s-1 ; q) \\
& -q^{(k-2)(m+s)-j+i-2} D_{k-1, k-i}(j-i+2 ; k-2 ; m+s-1 ; q)
\end{aligned}
$$

(by Lemma 1).

Recalling that $j(s, 0)+1=j(s, 1)$ and that $n=(k-1) m+r$, we note that the negative term of the above sum (when substituted into (4.12)) produces a sum that cancels the third sum appearing in (4.12). Hence substituting the righthand side of (4.13) into the first sum in (4.12), we find that

$$
\begin{aligned}
& g_{k, i}(n ; q)-g_{k, i-1}(n ; q) \\
& =\sum_{\substack{s=-\infty \\
j=i(s, 0)}}^{\infty} q^{(n-j)(m+s+1)} \frac{\left(q^{n-j}\right)_{\infty}}{\left(q^{n+1}\right)_{\infty}} D_{k-1, i-1}(j ; k-3 ; m+s ; q) \\
& +\sum_{\substack{s=-\infty \\
j=j(s, 0)}}^{\infty} q^{(n-j)(m+s+1)+(k-2)(m+s)-i+i-2 \frac{\left(q^{n-j}\right)}{\left(q^{n+1}\right)_{\infty}}} \\
& \text { - } D_{k-1, k-i+1}(j-i+2 ; k-2 ; m+s-1 ; q) \\
& +\sum_{\substack{s=-\infty \\
j=j(s, 0)}}^{\infty} q^{(n-j)(m+s+1)+j} \frac{\left(q^{n-i+1}\right)_{\infty}}{\left(q^{n+1}\right)_{\infty}} D_{k-1, k-i}(j-i+1 ; k-2 ; m+s-1 ; q) \\
& -\sum_{\substack{s=-\infty \\
j=j(s, 1)}}^{\infty} q^{(n-i)(m+s+2)+m+s+1} \frac{\left(q^{n-j+1}\right)_{\infty}}{\left(q^{n+1}\right)_{\infty}} D_{k-1, i-1}(j-1 ; k-3 ; m+s ; q) \\
& +\sum_{s=-\infty}^{\infty} \sum_{\substack{t=2 ; \\
j=j(s, t)}}^{k-2} q^{(n-i)(m+s+2)+j} \frac{\left(q^{n-j+1}\right)_{\infty}}{\left(q^{n+1}\right)_{\infty}} D_{k-1, k-i}(j-i+1 ; t-1 ; m+s ; q) \\
& -\sum_{s=-\infty}^{\infty} \sum_{\substack{t=2: i \\
j=j(s, t)}}^{k-2} q^{(n-j)(m+s+2)+t(m+s+1)} \frac{\left(q^{n-i+1}\right)_{\infty}}{\left(q^{n+1}\right)_{\infty}} \\
& \cdot D_{k-1, i-1}(j-t ; k-2-t ; m+s ; q) \text {. }
\end{aligned}
$$

Recalling that $j(s, 0)+1=j(s, 1)$, we see that each term of the first sum in (4.14) equals $q^{n-j(s, 0)-1}$ times the corresponding term of the first sum. Consequently we can combine the first and fourth sums in (4.14), and we obtain 


$$
\begin{aligned}
& g_{k, i}(n ; q)-g_{k, i-1}(n ; q) \\
& =\sum_{\substack{s=-\infty \\
j=j(s, 0)}}^{\infty} q^{(n-j)(m+s+1) \frac{\left(q^{n-j-1}\right)_{\infty}}{\left(q^{n+1}\right)_{\infty}} D_{k-1, i-1}(j ; k-3 ; m+s ; q)} \\
& +\sum_{\substack{s=-\infty \\
j=j(s, 0)}}^{\infty} q^{(n-j)(m+s+1)+(k-2)(m+s)-j+i-2 \frac{\left(q^{n-j}\right)_{\infty}}{\left(q^{n+1}\right)_{\infty}}} \\
& \text { - } D_{k-1, k-i+1}(j-i+2 ; k-2 ; m+s-1 ; q) \\
& +\sum_{\substack{s=-\infty \\
j=j(s, 0)}}^{\infty} q^{(n-j)(m+s+1)+j} \frac{\left(q^{n-j+1}\right)_{\infty}}{\left(q^{n+1}\right)_{\infty}} D_{k-1, k-i}(j-i+1 ; k-2 ; m+s-1 ; q) \\
& +\sum_{s=-\infty}^{\infty} \sum_{\substack{t=2 ; \\
j=j(s, t)}}^{k-2} q^{(n-j)(m+s+2)+j\left(q^{n-j+1}\right)_{\infty}} \frac{D_{k-1, k-i}(j-i+1 ; t-1 ; m+s ; q)}{\left(q^{n+1}\right)_{\infty}} \\
& -\sum_{s=-\infty}^{\infty} \sum_{\substack{t=2 ; \\
j=j(s, t)}}^{k-2} q^{(n-j)(m+s+2)+t(m+s+1) \frac{\left(q^{n-j+1}\right)_{\infty}}{\left(q^{n+1}\right)_{\infty}}} \\
& \text { - } D_{k-1, i-1}(j-t ; k-2-t ; m+s ; q) .
\end{aligned}
$$

We now assert that for $2 \leqslant h \leqslant k-1$,

$$
\begin{aligned}
& g_{k, i}(n ; q)-g_{k, i-1}(n ; q) \\
& =\sum_{\substack{s=-\infty \\
j=j(s, 0)}}^{\infty} q^{(n-j)(m+s+1)\left(q^{n-j-h+1}\right)_{\infty}} D_{k-1, i-1}(j ; k-h-1 ; m+s ; q) \\
& +\Sigma^{*}+\Sigma_{3}+\Sigma_{4}+\cdots+\sum_{n+1} \\
& +\sum_{s=-\infty}^{\infty} \sum_{\substack{t=h ; \\
j=j(s, t)}}^{k-2} q^{(n-j)(m+s+2)+j} \frac{\left(q^{n-j+1}\right)_{\infty}}{\left(q^{n+1}\right)_{\infty}} D_{k-1, k-i}(j-i+1 ; t-1 ; m+s ; q) \\
& -\sum_{s=-\infty}^{\infty} \sum_{\substack{t=h ; \\
j=j(s, t)}}^{k-2} q^{(n-j)(m+s+2)+t(m+s+1)} \frac{\left(q^{n-j+1}\right)_{\infty}}{\left(q^{n+1}\right)_{\infty}} \\
& \text { - } D_{k-1, i-1}(j-t ; k-2-t ; m+s ; q)
\end{aligned}
$$

where

$$
\Sigma^{*}=\sum_{\substack{s=-\infty \\ j=j(s, 0)}}^{\infty} q^{(n-j)(m+s+1)+j} \frac{\left(q^{n-j+1}\right)_{\infty}}{\left(q^{n+1}\right)_{\infty}} D_{k-1, k-i}(j-i+1 ; k-2 ; m+s-1 ; q),
$$

and 
54

G. E. ANDREWS

$$
\begin{aligned}
\sum_{e}=\sum_{\substack{s=-\infty \\
j=j(s, 0)}} q^{(n-j+k-e+1)(m+s+1)-j-k+i} \frac{\left(q^{n-j-e+3}\right)_{\infty}}{\left(q^{n+1}\right)_{\infty}} \\
\cdot D_{k-1, k-i+1}(j-i+e-1 ; e-3 ; m+s ; q) .
\end{aligned}
$$

When $h=2$, we see that (4.16) reduces to (4.15), since by Lemma 2

$$
\begin{array}{r}
\sum_{3}=\sum_{\substack{s=-\infty \\
j=j(s, 0)}}^{\infty} q^{(n-j)(m+s+1)+(k-2)(m+s)-j+i-2} \frac{\left(q^{n-j}\right)_{\infty}}{\left(q^{n+1}\right)_{\infty}} \\
\cdot D_{k-1, k-i+1}(j-i+2 ; k-2 ; m+s-1 ; q) .
\end{array}
$$

We proceed to establish (4.16) by induction on $h$. As we have remarked (4.16) is valid for $h=2$. We now assume that (4.16) is true for a fixed $h$, with $2 \leqslant h<k-1$, and we shall show that this implies its truth for $h+1$.

We start by transforming the $D_{k-1, l-1}(j ; k-h-1 ; m+s ; q)$ in the same manner that $D_{k-1, t-1}(j ; k-2 ; m+s ; q)$ was transformed in (4.13). Namely

$$
\begin{aligned}
& D_{k-1, t-1}(j ; k-h-1 ; m+s ; q) \\
& \quad=D_{k-1,1-1}(j ; k-h-2 ; m+s ; q) \\
& \quad+q^{(k-h-1)(m+s+1)-j-k+i} D_{k-1, k-i+1}(j+h+1-i ; h-1 ; m+s ; q) \\
& \quad-q^{(k-h-1)(m+s+1)-1-k+i} D_{k-1, k-1}(j+h+1-i ; h-1 ; m+s ; q) .
\end{aligned}
$$

(4.17)

Substituting the right-hand side of (4.17) into the first sum appearing in (4.16), we see that$$
g_{k, i}(n ; q)-g_{k, i-1}(n ; q)
$$$$
=\sum_{\substack{s=-\infty ; \\ j=j(s, 0)}}^{\infty} q^{(n-j)(m+s+1)\left(q^{n-i-h+1}\right)_{\infty}} D_{k-1, i-1}(j ; k-h-2 ; m+s ; q)
$$

$$
\begin{aligned}
& +\sum_{\substack{s=-\infty \\
j=i(s, 0)}}^{\infty} q^{(n-i)(m+s+1)+(k-h-1)(m+s+1)-j-k+i \frac{\left(q^{n-j-h+1}\right)_{\infty}}{\left(q^{n+1}\right)_{\infty}}} \\
& \cdot D_{k-1, k-i+1}(j+h+1-i ; h-1 ; m+s ; q) \\
& -\sum_{\substack{s=-\infty \\
j=j(s, 0)}}^{\infty} q^{(n-i)(m+s+1)+(k-h-1)(m+s+1)-j-k+i} \frac{\left(q^{n-i-h+1}\right)_{\infty}}{\left(q^{n+1}\right)_{\infty}} \\
& \quad \cdot D_{k-1, k-i}(j+h+1-i ; h-1 ; m+s ; q)+\sum^{*}+\sum_{3}+\sum_{4}+\cdots+\sum_{n+1}
\end{aligned}
$$




$$
\begin{aligned}
& +\sum_{\substack{s=-\infty, i \\
i=j(s, h)}}^{\infty} q^{(n-j)(m+s+2)+j \frac{\left(q^{n-j+1}\right)_{\infty}}{\left(q^{n+1}\right)_{\infty}} D_{k-1, k-i}(j-i+1 ; h-1 ; m+s ; q)} \\
& -\sum_{\substack{s=-\infty \\
i=j(s, h)}}^{\infty} q^{(n-j)(m+s+2)+h(m+s+1) \frac{\left(q^{n-j+1}\right)_{\infty}}{\left(q^{n+1}\right)_{\infty}}} \\
& +\sum_{s=-\infty}^{\infty} \sum_{\substack{t=h+1: \\
j=j(s, t)}}^{k-2} q_{k-1, k-i}^{(n-j)(m+s+2)+j} \frac{\left(q^{n-j+1}\right)_{\infty}}{\left(q^{n+1}\right)_{\infty}} D_{k-1, k-i}(j-i+1 ; t-1 ; m+s ; q) \\
& -\sum_{s=-\infty}^{\infty} \sum_{\substack{t=h+1 \\
j=j(s, t)}}^{k-2} q^{(n-j)(m+s+2)+t(m+s+1) \frac{\left(q^{n-j+1}\right)_{\infty}}{\left(q^{n+1}\right)_{\infty}}} \\
& \quad D_{k-1, i-1}(j-t ; k-2-t ; m+s ; q) .
\end{aligned}
$$

((4.18) continued)

Recalling that $j(s, h)=j(s, 0)+h$ and that $n=(k-1) m+r$, we see that the sum preceding $\Sigma^{*}$ in (4.18) cancels the sum following $\Sigma_{h+1}$ in (4.18); the second sum in (4.18) is $\Sigma_{h+2}$; finally we note that the third sum from the end of (4.18) is term by term $q^{n-j-h}$ times the corresponding term of the first sum. With all these observations taken into account, we see that (4.18) may be rewritten as

$$
\begin{aligned}
& \boldsymbol{g}_{k, i}(n ; q)-g_{k, i-1}(n ; q) \\
& =\sum_{\substack{s=-\infty \\
j=j(s, 0)}}^{\infty} q^{(n-j)(m+s+1)} \frac{\left(q^{n-j-h}\right)_{\infty}}{\left(q^{n+1}\right)_{\infty}} D_{k-1, i-1}(j ; k-h-2 ; m+s ; q) \\
& +\Sigma^{*}+\sum_{3}+\Sigma_{4}+\cdots+\sum_{n+1}+\sum_{n+2} \\
& +\sum_{s=-\infty}^{\infty} \sum_{\substack{t=n+1 \\
j=j(s, i)}}^{k-2} q^{(n-j)(m+s+2)+j} \frac{\left(q^{n-j+1}\right)_{\infty}}{\left(q^{n+1}\right)_{\infty}} D_{k-1, k-i}(j-i+1 ; t-1 ; m+s ; q) \\
& -\sum_{s=-\infty}^{\infty} \sum_{\substack{r=h+1 \\
j=j(s, t)}}^{k-2} q^{(n-j)(m+s+2)+t(m+s+1) \frac{\left(q^{n-j+1}\right)_{\infty}}{\left(q^{n+1}\right)_{\infty}}} \\
& \cdot D_{k-1, i-1}(j-t ; k-2-t ; m+s ; q) .
\end{aligned}
$$

Now (4.19) is just (4.16) with $h$ replaced by $h+1$. Consequently (4.16) is proved for $2 \leqslant h \leqslant k-1$. Thus setting $h=k-1$, we find that (4.16) reduces to 
(4.20)

$$
\begin{aligned}
g_{k, i}(n ; q) & -g_{n, i-1}(n ; q) \\
= & \sum_{\substack{s=-\infty \\
j=j(s, 0)}}^{\infty} q^{(n-j)(m+s+1)} \frac{\left(q^{n-j-k+2}\right)_{\infty}}{\left(q^{n+1}\right)_{\infty}} D_{k-1, i-1}(j ; 0 ; m+s ; q) \\
& +\sum^{*}+\sum_{3}+\sum_{4}+\cdots+\sum_{k}
\end{aligned}
$$

We now combine the first sum on the right-hand side of (4.20) (which we call $\Sigma^{\prime}$ ) with $\Sigma^{*}$. In $\Sigma^{*}$, we replace $s$ by $s+1$ (which replaces $j$ by $j+$ $k-1)$, and then we replace $D_{k-1, k-i}(j+k-i ; k-2 ; m+s ; q)$ by

$$
D_{k-1, k-i+1}(j+k-i ; k-2 ; m+s ; q)-q^{j+k-i} D_{k-1, i-1}(j ; k-2 ; m+s-1 ; q)
$$

(which is valid by Lemma 1).

Thus

$$
\begin{aligned}
& \sum^{*}=\sum_{\substack{s=-\infty \\
j=j(s, 0)}}^{\infty} q^{(n-j-k+1)(m+s+2)+j+k-1} \frac{\left(q^{n-j-k+2}\right)_{\infty}}{\left(q^{n+1}\right)_{\infty}} \\
& \cdot\left\{D_{k-1, k-i+1}(j+k-i ; k-2 ; m+s ; q)\right. \\
&\left.-q^{j+k-i} D_{k-1, i-1}(j ; k-2 ; m+s-1 ; q)\right\} .
\end{aligned}
$$

Recalling that $n=(k-1) m+r$ and that $D_{k-1, i-1}(j ; k-2 ; m+s-1 ; q)=$ $D_{k-1, i-1}(j ; 0 ; m+s ; q)$, we see that

$$
\begin{aligned}
\Sigma^{*}=\sum_{\substack{s=-\infty \\
j=j(s, 0)}}^{\infty} q^{(n-j-k+1)(m+s+2)+j+k-1} \frac{\left(q^{n-j-k+2}\right)_{\infty}}{\left(q^{n+1}\right)_{\infty}} \\
\cdot D_{k-1, k-i+1}(j+k-i ; k-2 ; m+s ; q)-\Sigma^{\prime} .
\end{aligned}
$$

Thus if we replace $\Sigma^{*}+\Sigma^{\prime}$ by the above sum in (4.20), we see that

$$
\begin{aligned}
& g_{k, i}(n ; q)-g_{k, i-1}(n ; q) \\
&=\sum_{\substack{s=-\infty \\
j=j(s, 0)}}^{\infty} q^{(n-j-k+1)(m+s+2)+j+k-1} \frac{\left(q^{n-j-k+2}\right)_{\infty}}{\left(q^{n+1}\right)_{\infty}} \\
& \quad \cdot D_{k-1, k-i+1}(j+k-i ; k-2 ; m+s ; q)+\sum_{3}+\sum_{4}+\cdots+\sum_{k} .
\end{aligned}
$$

Replacing $s$ by $s-1$ in the first term on the right-hand side of (4.21) (which replaces $j$ by $j-k+1$ ), we see that 


$$
\begin{aligned}
& g_{k, i}(n ; q)-g_{k, i-1}(n ; q) \\
& =\sum_{\substack{s=-\infty \\
j=j(s, 0)}}^{\infty} q^{(n-j)(m+s+1)+j} \frac{\left(q^{n-j+1}\right)_{\infty}}{\left(q^{n+1}\right)_{\infty}} D_{k-1, k-i+1}(j+k-i ; k-2 ; m+s-1 ; q) \\
& +\sum_{\substack{s=-\infty \\
j=j(s, 0)}}^{\infty} q^{(n-j)(m+s+1)+(k-2)(m+s)-j+i-2 \frac{\left(q^{n-j}\right)_{e}}{\left(q^{n+1}\right)_{\infty}}} \\
& \text { - } D_{k-1, k-i+1}(j-i+2 ; k-2 ; m+s-1 ; q) \\
& +\sum_{s=-\infty}^{\infty} \sum_{\substack{t=2 ; \\
j=j(s, 0)}}^{k-2} q^{(n-j+k-t-1)(m+s+1)-j-k+i \frac{\left(q^{n-j-t+1}\right)_{\infty}}{\left(q^{n+1}\right)_{\infty}}} \\
& \text { - } D_{k-1, k-i+1}(j+t+1-i ; t-1 ; m+s ; q) .
\end{aligned}
$$

Our object now is to identify the right-hand side of (4.22) with

$$
q^{n}\left(1-q^{n}\right) \cdots\left(1-q^{n-i+2}\right) g_{k, k-i+1}(n-i+1 ; q) \text {. }
$$

To do this we utilize (4.7) replacing $i$ by $k-i+1, n$ by $n-i+1$, and $i$ by $(k-1) s+t-r$ where $-\infty<s<\infty, 0 \leqslant t \leqslant k-2$. Hence

$$
\begin{aligned}
& q^{n}\left(1-q^{n}\right) \cdots\left(1-q^{n-i+2}\right) g_{k, k-i+1}(n-i+1 ; q) \\
& =q^{n} \sum_{\substack{s=-\infty \\
j=(k-1) s+t-r}}^{\infty} \sum_{\substack{t=0 \\
s-2}} q^{(n-i+1-j)\left(m+s+1+\left[\frac{t-1}{k-1}\right]\right)} \frac{\left(q^{n-i-j+2}\right)_{\infty}}{\left(q^{n+1}\right)_{\infty}} \\
& \cdot D_{k-1, k-i+1}\left(j, \eta(t-2) ; m+s+\left[\frac{t-2}{k-1}\right] ; q\right) \\
& =\sum_{s=-\infty}^{\infty} q^{n+(n-i+1-(k-1) s+r)(m+s)} \frac{\left(q^{n-(k-1) s-i+r+2}\right)_{\infty}}{\left(q^{n+1}\right)_{\infty}} \\
& \text { - } D_{k-1, k-i+1}((k-1) s-r ; k-2 ; m+s-1 ; q) \\
& +\sum_{s=-\infty}^{\infty} q^{n+(n-i+1-(k-1) s-1+r)(m+s+1) \frac{\left(q^{n-i-j+2}\right)_{\infty}}{\left(q^{n+1}\right)_{\infty}}} \\
& \cdot D_{k-1, k-i+1}(j ; k-2 ; m+s-1 ; q) \\
& +\sum_{s=-\infty}^{\infty} \sum_{t=2}^{k-2} q^{n+(n-i+1-(k-1) s-t+r)(m+s+1) \frac{\left(q^{n-i-j+2}\right)_{\infty}}{\left(q^{n+1}\right)_{\infty}}} \\
& \cdot D_{k-1, k-i+1}(j ; t-1 ; m+s ; q) \text {. }
\end{aligned}
$$


Recalling that $n=(k-1) m+r$ and that $j(s, 0)=(k-1) s+i-r-1$, we see that the right-hand side of (4.23) is identical term by term with the right-hand side of (4.22). Consequently for $2 \leqslant i \leqslant k$, we see that

$$
\begin{aligned}
g_{k, i}(n ; q) & -g_{k, t-1}(n ; q) \\
= & q^{n}\left(1-q^{n}\right) \cdots\left(1-q^{n-i+2}\right) g_{k, k-t+1}(n-i+1 ; q) .
\end{aligned}
$$

Thus we have established that the $g_{k, i}(n ; q)$ satisfy $(4.1),(4.2)$ and (4.3). Therefore by the remark following (4.3),

$$
G_{k, i}(n ; q)=g_{k, i}(n ; q),
$$

and this is precisely Theorem 1.

The proof of Theorem 2 now follows directly from Theorem 1 .

$$
\begin{aligned}
\sum_{n=0}^{\infty} A_{k, i}(n) q^{n}= & \prod_{\substack{n=1 ; \\
n \neq 0, \pm i\left(\bmod ^{\prime} 2 k+1\right)}}^{\infty}\left(1-q^{n}\right)^{-1} \\
= & \left.Q_{k, i}(1 ; q) \quad \text { (by Jacobi's identity }[8, \mathrm{p} .282]\right) \\
= & \sum_{n=0}^{\infty} G_{k, i}(n ; q) /(q)_{n} \\
= & \sum_{n=0}^{\infty} \sum_{j=0}^{n} \frac{q^{(n-j)\left[\frac{n+j+2 k-i-2}{k-1}\right]}}{(q)_{n-j}} \\
& \cdot D_{k-1, i}\left(j ; n(n+j+k-i-2) ;\left[\frac{n+j+k-i-2}{k-1}\right] ; q\right) .
\end{aligned}
$$

Recalling-the definition of the $(k, i)$-cutoff of a partition with $n$ parts, we see that

$$
q^{(n-j)[(n+j+2 k-i-2) /(k-1)]} /(q)_{n-j}
$$

generates partitions with $n-j$ parts each $\geqslant[(n+j+2 k-i-2) /(k-1)]$, and

- (4.27) $D_{k-1, i}(j ; \eta(n+j+k-i-2) ;[(n+j+k-i-2) /(k-1)] ; q)$

generates partitions with $j$ parts subject to the conditions defining the $D_{k-1, i}$ including the requirement that each part is $\leqslant[(n+j+k-i-2) /(k-1)]$. Thus the product of (4.26) and (4.27) generates partitions with $n$ parts and $(k, i)$-cutoff $=[(n+j+k-i-2) /(k-1)]$ subject to the condition that the $j$ parts $\leqslant$ the $(k, i)$-cutoff taken together form a partition of the type enumerated by the partition generating function appearing in (4.27). The fact that 
$[(n+j+k-i-2) /(k-1)]$ is indeed the $(k, i)$-cutoff of the partitions generated is somewhat subtle. It is clear that there are $n$ parts in each such partition and furthermore the largest $n-j$ parts are all $\geqslant[(n+j+2 k-i-2) /(k-1)]$, but it is not immediately obvious that some smaller $j$ will not fill the bill in some instances. This latter eventuality is impossible since by the difference conditions defining $D_{k-1, t}$, we see that the $r$ th largest part $b_{n-j+r}$ of the final $j$ parts is $\leqslant[(n+j+k-i-2) /(k-1)]-2[(r-1) /(k-1)]$. Now if $(r-1)=c \cdot(k-1)+d, 0 \leqslant d<k-1$, then if $c>0$,

$$
\begin{aligned}
b_{n-j+r} & \leqslant\left[\frac{n+j+k-i-2}{k-1}\right]-2\left[\begin{array}{l}
r-1 \\
k-1
\end{array}\right]=\left[\frac{n+j+k-i-2-2 c(k-1)}{k-1}\right] \\
& <\left[\frac{n+(j-c(k-1)-d)+2 k-i-2}{k-1}\right]=\left[\frac{n+(j-r)+2 k-i-2}{k-1}\right],
\end{aligned}
$$

since

$$
\begin{aligned}
(n+(j-c(k-1)-d)+ & 2 k-i-2)-(n+j+k-i-2-2 c(k-1)) \\
& =k+c(k-1)-d \\
& \geqslant k-1+k-d>k-1 .
\end{aligned}
$$

If $r-1<k-1$, then $c=0$ and the above argument is inadequate; however, now we note that if $\lambda=[(n+j+k-i-2) /(k-1)]$, then

$$
\begin{aligned}
n+j+k-i-2 & \geqslant \lambda \cdot(k-1)+\eta(n+j+k-i-2) \\
& =\lambda \cdot(k-1)+\eta \quad \text { (for brevity). }
\end{aligned}
$$

Thus for $1 \leqslant r \leqslant \eta$,

$$
\begin{aligned}
b_{n-i+r} & \leqslant[(n+j+k-i-2) /(k-1)]=\lambda<\lambda+[(\eta+k-r) /(k-1)] \\
& =[(\lambda(k-1)+\eta+k-r) /(k-1)] \\
& \leqslant[(n+(j-r)+2 k-i-2) /(k-1)] .
\end{aligned}
$$

For $\eta<r<k-1$,

$$
\begin{aligned}
b_{n-1+r} & \leqslant\left[\frac{n+j+k-i-2}{k-1}\right]-1=\lambda-1<\lambda+\left[\frac{n+k-r}{k-1}\right] \\
& \leqslant\left[\frac{n+(j-r)+2 k-i-2}{k-1}\right] .
\end{aligned}
$$

Thus we see that $[(n+j+k-i-2) /(k-1)]$ is truly the $(k, i)$-cutoff of the partitions generated.

Summing over all nonnegative $n$ and over all $j$ with $0 \leqslant j \leqslant n$, we find that 
Table 1. Partitions of $n$ enumerated by:

\begin{tabular}{|c|c|c|c|}
\hline$n$ & $A_{3,3}(n)$ & $B_{3,3}(n)$ & $H_{3,3}(n)$ \\
\hline 1 & 1 & 1 & 1 \\
\hline \multirow[t]{2}{*}{2} & 2 & 2 & 2 \\
\hline & $1+1$ & $1+1$ & $1+1$ \\
\hline \multirow[t]{2}{*}{3} & $2+1$ & 3 & 3 \\
\hline & $1+1+1$ & $2+1$ & $2+1$ \\
\hline \multirow[t]{3}{*}{4} & $2+2$ & 4 & 4 \\
\hline & $2+1+1$ & $3+1$ & $3+1$ \\
\hline & $1+1+1+1$ & $2+2$ & $2+2$ \\
\hline \multirow[t]{4}{*}{5} & 5 & 5 & 5 \\
\hline & $2+2+1$ & $4+1$ & $4+1$ \\
\hline & $2+1+1+1$ & $3+2$ & $3+2$ \\
\hline & $1+1+1+1+1$ & $3+1+1$ & $2+2+1$ \\
\hline \multirow[t]{6}{*}{6} & 6 & 6 & 6 \\
\hline & $5+1$ & $5+1$ & $5+1$ \\
\hline & $2+2+2$ & $4+2$ & $4+2$ \\
\hline & $2+2+1+1$ & $3+3$ & $3+3$ \\
\hline & $2+1+1+1+1$ & $4+1+1$ & $2+2+2$ \\
\hline & $1+1+1+1+1+1$ & $3+2+1$ & $3+2+1$ \\
\hline \multirow[t]{7}{*}{7} & $6+1$ & 7 & 7 \\
\hline & $5+2$ & $6+1$ & $6+1$ \\
\hline & $5+1+1$ & $s+2$ & $5+2$ \\
\hline & $2+2+2+1$ & $4+3$ & $4+3$ \\
\hline & $2+2+1+1+1$ & $5+1+1$ & $3+3+1$ \\
\hline & $2+1+1+1+1+1$ & $4+2+1$ & $4+2+1$ \\
\hline & $1+1+1+1+1+1+1$ & $3+3+1$ & $3+2+2$ \\
\hline \multirow[t]{10}{*}{8} & 8 & 8 & 8 \\
\hline & $6+2$ & $7+1$ & $7+1$ \\
\hline & $6+1+1$ & $6+2$ & $6+2$ \\
\hline & $5+2+1$ & $5+3$ & $5+3$ \\
\hline & $5+1+1+1$ & $4+4$ & $4+4$ \\
\hline & $2+2+2+2$ & $6+1+1$ & $5+2+1$ \\
\hline & $2+2+2+1+1$ & $5+2+1$ & $4+3+1$ \\
\hline & $2+2+1+1+1+1$ & $4+3+1$ & $4+2+2$ \\
\hline & $2+1+1+1+1+1+1$ & $4+2+2$ & $3+3+2$ \\
\hline & $1+1+1+1+1+1+1+1$ & $3+3+1+1$ & $2+2+2+2$ \\
\hline
\end{tabular}




$$
\begin{aligned}
& \sum_{n=0}^{\infty} \sum_{j=0}^{n} \frac{q^{(n-j)\left[\frac{n+j+2 k-i-2}{k-1}\right]}}{(q)_{n-j}} \\
& \quad \cdot D_{k-1, i}\left(j ; \eta(n+j+k-i-2) ;\left[\frac{n+j+k-i-2}{k-1}\right] ; q\right) \\
& \quad=\sum_{n=0}^{\infty} H_{k, i}(n) q^{n} .
\end{aligned}
$$

Comparing (4.25) with (4.28), we deduce that

$$
\sum_{n=0}^{\infty} A_{k, i}(n) q^{n}=\sum_{n=0}^{\infty} H_{k, i}(n) q^{n}
$$

Therefore $A_{k, i}(n)=H_{k, i}(n)$ for each $n$ and Theorem 2 is proved.

We shall now show that Theorem 2 contains (1.6) and (1.7) (the RogersRamanujan identities) as special cases.

COROLlaRY 1. Identity (1.6) holds.

Proof. Set $k=i=2$ in Theorem 2. Noting that $D_{1,2}(j ; m ; N ; q)=1$, we see that the empty partition of zero is the only partition generated by this function. Thus the only partitions enumerated by $H_{2,2}(n)$ are those in which all the parts are $\geqslant$ the number of parts (see Definition 3 with $j=0$ ).

Hence by Theorem 2, $A_{2,2}(n)=H_{2,2}(n)$ which is now clearly (1.6).

COROLlary 2. Identity (1.7) holds.

Proof. Set $k=2, i=1$. Again we see that $D_{1,1}(j ; m ; N ; q)=1$. Thus as before the partitions enumerated by $H_{2,1}(n)$ are those in which all parts exceed the number of parts. Thus $A_{2,1}(n)=H_{2,1}(n)$ implies (1.7).

Finally we provide a short table related to Gordon's theorem (1.5) and Theorem 2.

Definition 7. Let $B_{k, i}(n)$ denote the number of partitions of $n$ of the form $n=b_{1}+b_{2}+\cdots+b_{s}$, where $b_{j} \geqslant b_{j+1}, b_{j}-b_{j+k-1} \geqslant 2$, and where 1 appears as a summand at most $i-1$ times.

As we observe from Table 1 , the correspondence between the partitions enumerated by $B_{3,3}(n)$ and $H_{3,3}(n)$ may be refined to a correspondence between partitions with the same number of summands. Such a refined correspondence is easily proved by observing that if $B_{k, i}(n ; m)\left(\operatorname{resp} . H_{k, i}(n ; m)\right)$ denotes the number of partitions enumerated by $B_{k, i}(n)$ (resp. $\left.H_{k, i}(n)\right)$ with $m$ summands, then 


$$
\begin{aligned}
\sum_{n=0}^{\infty} \sum_{m=0}^{\infty} B_{k, i}(n ; m) z^{m} q^{n} & =Q_{k, i}(z ; q) \quad \text { (by [3, equations (2.3) and (2.7)]) } \\
& =\sum_{n=0}^{\infty} G_{k, i}(n ; q) z^{n} /(q)_{n}=\sum_{n=0}^{\infty} \sum_{m=0}^{\infty} H_{k, i}(n ; m) z^{m} q^{n} .
\end{aligned}
$$

5. Formulae for $G_{3,3}(n ; q)$. As the importance of the Alder polynomials has teen clearly established by Theorems 1 and 2, we point out in this section that there are various interesting formulae for the $G_{k, i}(n ; q)$ when $k$ is small.

Of course as Alder pointed out, $G_{2, i}(n ; q)=q^{n^{2}+(2-i) n}$ for $i=1,2$. Less well known are formulae for $G_{k, i}(n ; q)$ with $k \geqslant 3$.

L. Carlitz [6, equation (9), p. 519] has proved that

$$
G_{k, k}(n ; q)=\sum_{0<k s \leqslant n}(-1)^{s} \frac{(q)_{n}}{(q)_{s}(q)_{n-k s}} q^{1 / 2 s(s-1)+s n}\left(1-q^{s}+q^{n-k s+s}\right) .
$$

Carlitz's derivation of (5.1) is made by comparing coefficients of $z^{n}$ in

$$
Q_{k, k}(z ; q)=\sum_{n=0}^{\infty} G_{k, k}(n ; q) z^{n} /(q)_{n} .
$$

Obviously by considering $Q_{k, i}(z ; q)$ instead of $Q_{k, k}(z ; q)$, we see that we could derive similar formulae for $G_{k, i}(n ; q)$.

By comparing coefficients of $x^{n}$ in $(5.10)$ of $[4$, p. 443], we see that

$$
G_{3,3}(n ; q)=q^{1 / 2 n(n+1)} \sum_{0<2 m<p} q^{-m}\left(\begin{array}{c}
p \\
2 m
\end{array}\right)_{q}\left(q ; q^{2}\right)_{m},
$$

where $(r)_{q}=(q)_{r} /(q)_{s}(q)_{r-s}$. Similar results can be obtained from (5.11) of $\left[4\right.$, p. 443] for $G_{3,2}(n ; q)$ and from $(5.12)$ of $\left[4\right.$, p. 443] for $G_{3,1}(n ; q)$.

Singh [10, equation (3.7)] also has obtained formulae for the Alder polynomials. In particular for $k=i=3$, Singh's formula reduces to

$$
G_{3,3}(n ; q)=q^{n^{2}} \sum_{0<2 s<n}\left(\begin{array}{l}
n \\
2 s
\end{array}\right)_{q}\left(q^{s+1}\right)_{s} q^{2 s^{2}-2 s n} .
$$

Also in this vein we note that Theorem 1 may be used to produce a further formula of this type for $k=3$. We note that

$$
D_{2,2}(j ; 1 ;[(n+j-2) / 2] ; q)=q^{j^{2}}\left(\begin{array}{c}
{[(n-j) / 2]} \\
j
\end{array}\right)_{q} \text {. }
$$

This is easily seen to be the case if we recall $[13$, p. 4 of Collected mathematical papers, Volume IV] that $\left(\begin{array}{c}m \\ r\end{array}\right)_{q}$ is the generating function for partitions with at most $r$ parts and with largest part $\leqslant m-r$. Using this fact, (5.5) is verified in 
the same manner in which one usually derives the generating function for $B_{2,2}(n)$ [8, pp. 290-291]. Hence by Theorem 1,

$$
G_{3,3}(n ; q)=\sum_{s \geq 0} q^{(n-j)[(n+j+1) / 2]+j^{2}}\left(\begin{array}{c}
{[(n-j) / 2]} \\
j
\end{array}\right)_{q}\left(q^{n-j+1}\right)_{j}
$$

We have now exhibited 4 different formulae ((5.1), (5.3), (5.4) and (5.6)) for $G_{3,3}(n ; q)$. It would be most interesting to know how these formulae fit into the general theory of basic hypergeometric series, and how the Alder polynomials generally fit into this theory. Certainly Singh's [10], [11], [12] and Carlitz's [6] papers make a significant contribution to such a study.

6. Conclusion. The results discussed in this paper suggest that Alder-type polynomials may be important in a general situation. Let us briefly suggest such a general situation, referring to [5] for notation and definitions.

Let $C$ be a partition ideal with

$$
f_{C}(z ; q)=\sum_{\left\{f_{i}\right\} \in C} z^{\Sigma f_{i}} q^{\Sigma f_{i} i}
$$

Define the Alder function $G_{C}(n ; q)$ by

$$
f_{C}(z ; q)=\sum_{n=0}^{\infty} G_{C}(n ; q) z^{n} /(q)_{n} .
$$

What conditions are required on $C$ in order that $G_{C}(n ; q)$ be a polynomial for each $n$ ? What conditions on $C$ are necessary in order that $G_{C}(n ; q)$ have an expansion like that given in Theorem 1 for $G_{k, i}(n ; q)$ ?

\section{REFERENCES}

1. H. L. Alder, Generalizations of the Rogers-Ramanujan identitles, Pacific J. Math. 4 (1954), 161-168. MR 15, 856.

2. - Partition identities-from Euler to the present, Amer. Math. Monthly 76 (1969), 733-746. MR 41 \#8366.

3. G. E. Andrews, An analytic proof of the Rogers-Ramanujan-Gordon identities, Amer. J. Math. 88 (1'966), 844-846. MR 34 \#2478.

4. - On q-difference equations for certain well-poised basic hypergeometric series, Quart. J. Math. Oxford Ser. (2) 19 (1968), 433-447. MR 38 \#6112.

5. - Partition identities, Advances in Math. 9 (1972), 10-51.

6. L. Carlitz, Note on Alder's polynomials, Pacific J. Math. 10 (1960), 517-519. MR 22 \#3713.

7. B. Gordon, A combinatortal generalization of the Rogers-Ramanujan identities, Amer. J. Math. 83 (1961) 393-399. MR 23 \#A809.

8. G. H. Hardy and E. M. Wright, An introduction to the theory of numbers, 4th ed., Oxford Univ. Press, Oxford, 1960.

9. A. Selberg, Über einige arithmetische Identitäten, Avhandlinger Norske Akad. 1936, no. 8.

10. V. N. Singh, Certain generalized hypergeometric identities of the RogersRamanujan type, Pacific J. Math. 7 (1957), 1011-1014. MR 19, 410. 
11. Certain generalized hypergeometric identities of the Rogers-Ramanujan type. (II), Pacific J. Math. 7 (1957), 1691-1699. MR 20 \#1803.

12. A note on the computation of Alder's polynomials, Pacific J. Math. 9 (1959), 271-275. MR 21 \#7318.

13. J. J. Sylvester, A constructive theory of partitions arranged in three acts, an interact and an exodion, Amer. J. Math. 5 (1882), 251 -330; ibid. 6 (1884), 334-336 (or pp. 1-83 of the Collected mathematical papers of James Joseph Sylvester. Vol. 4, Cambridge Univ. Press, Cambridge, 1912).

DEPARTMENT OF MATHEMATICS, PENNSYLVANIA STATE UNIVERSITY, UNIVERSITY PARK, PENNSYLVANIA 16802 